\title{
Electrical Characterization of Polyester Reinforced by Carbon Black Particles
}

\author{
Omed Gh. Abdullah, Gelas M. Jamal, Dana A. Tahir, and Salah R. Saeed
}

\begin{abstract}
Polymer matrix composite, based on Polyester reinforced with $(0,6,12$, and 18$) \%$ of carbon black has been prepared using solution cast technique. The dielectric constant and $a c$ electrical conductivity of the samples are investigated in the frequency range $\left(0.5-10^{3}\right) \mathrm{KHz}$ and within the temperature range $(26-80)^{\circ} \mathrm{C}$. Dielectric constant and loss tangent decreases with increasing frequency and increase with increasing temperature. The $a c$ electrical conductivity $\sigma_{a c}$ for all samples were found to be weak frequency dependent at low frequency, however vary with frequency as a power law $\omega^{s}$ at higher frequency range. The exponential factor $s$ found to be in the range $0.65<s<0.77$. The weakly decreases of $s$ with increasing temperature, could be attributed that higher temperature, high mobility of free charges make them more frequency independent conductivity. From the temperature dependence of $d c$ electrical conductivity, the activation energy at each frequency was calculated using the Arrhenius relation. The variation of the activation energy as a function of carbon black contents, and frequency is also discussed.
\end{abstract}

Index Terms-Ac conductivity, de conductivity, dielectric constant, polyester, carbon black.

\section{INTRODUCTION}

Polymers have received much experimental attention in an attempt to synthesize organic polymers alternative to conventional inorganic materials, due to their unique properties, such as low density, ability to form intricate shapes, versatile electric properties and low manufacturing cost [1]. The dielectric behavior of polymer films is of considerable interest due to their applications for insulation, isolation and microelectronics [2]. The dielectric constant and the loss factors are the most convenient and sensitive methods for studying the polymer structure [3].

The electrical conductivity of insulating polymers materials can be enhanced several orders of magnitude by incorporating some conducting filler in it [4]. Due to the low cost and good electrical conductivity, carbon black (CB)-filled polymers have been widely used for several decades in many applications [5]. In addition, carbon black characterize by a good absorption performance in high frequency bands [6]. The dielectric radar absorbing material has been made by adding some conductive powders like carbon black or silver particle to the matrix resin in order to induce the dielectric loss by enhancing the electrical

Manuscript received August 24, 2011.

Omed Gh. Abdullah, is with the, Physics Department, Faculty of Science and Science Education, University of Sulaimani, Sulaimani, P.O.Box: 342, Iraq, (omed.abdullah@univsul.net).

Gelas M. Jamal, Dana A. Tahir, and Salah R. Saeed are with the, Physics Department, Faculty of Science and Science Education, University of Sulaimani, Sulaimani-Iraq, (email: gelas.jamal@univsul.net, dana.tahir@univsul.net, salah.saeed@univsul.net). conductivity of the mixture. Among them, carbon black is widely used due to its good absorption performance in high frequency bands [6].

In order to reveal the nature of the polymer and its molecular structure, the dielectric properties, $a c$ electrical conductivity, and loss tangent of Polyester reinforced with carbon black has been investigated at different temperatures and frequencies. The studies also include the role of carbon black addition in varying concentrations to a polymer matrix.

\section{THEORY}

In an alternating $a c$ field, the dielectric constant is a complex quantity $\varepsilon^{*}$, which consist of two combinations, the real part which is known as the dielectric constant $\varepsilon^{\prime}$ and imaginary part called the dielectric loss or dissipation factor $\varepsilon^{\prime \prime}[7]$. The complex dielectric constant $\varepsilon^{*}$ as a function of angular frequency $(\omega)$ of the measuring electric field can be represented as [8]:

$$
\varepsilon^{*}=\varepsilon^{\prime}(\omega)-i \varepsilon^{\prime \prime}(\omega)
$$

The dielectric constant $\varepsilon^{\prime}$ of sample is evaluated from the capacitance $C_{s}$ measured using equation:

$$
\varepsilon^{\prime}=\frac{C_{S} d}{\varepsilon_{o} A}
$$

where $\varepsilon_{o}$ is constant of free space, $A$ and $d$ are the effective cross-sectional area and thickness of the sample respectively.

The dielectric loss factor $\varepsilon^{\prime \prime}$ can be calculated from measurement value $a c$ electrical conductance by [9]:

$$
\varepsilon^{\prime \prime}=\frac{\sigma_{a c}}{\varepsilon_{o} \omega}
$$

here $\sigma_{a c}$ represents the $a c$ - electrical conductivity of the polymer sample which arises from the motion of charge carriers through the polymer.

The general formula of $\sigma_{a c}$ as a function of polymer resistance $R_{s}$ is given by [10]:

$$
\sigma_{a c}=\frac{d}{R_{S} A}
$$

The values of $\varepsilon^{\prime}$ and $\varepsilon^{\prime \prime}$ can be used to calculate the loss tangent $\tan (\delta)$ using this equation:

$$
\varepsilon^{\prime \prime}=\varepsilon^{\prime} \tan (\delta)
$$

where $\delta$ is the phase angle between the electric field and the polarization of the dielectric.

In general the complex electrical conductivity $\sigma^{*}$ was written as: 


$$
\sigma^{*}=\sigma^{\prime}(\omega)+i \sigma^{\prime \prime}(\omega)
$$

where $\sigma^{\prime}$ and $\sigma^{\prime \prime}$ are the real and imaginary parts of the electrical conductance. The real part of conductivity $\sigma^{\prime}$ has been often analyzed separated in two different components:

$$
\sigma^{\prime}=\sigma_{d c}+\sigma_{a c}
$$

The $d c$ electrical conductivity $\sigma_{d c}$ usually described by hopping models of electrons near the Fermi level [11]. The term hopping refers to the sudden displacement of charge carriers from one position to another neighboring site and in general includes both jumps over a potential barrier and quantum mechanical tunneling [12].

In the polymer material the frequency and temperature dependent conductivity is caused by the hopping of the charge carriers in the localized state and also due to the excitation of charge carriers to upper states in the conduction band $[13,14]$. The conductivity obeys the empirical universal power law relation [15]:

$$
\sigma^{\prime}(\omega, T)=\sigma_{d c}(T)+A \omega^{s}
$$

where $A$ is dependent on temperature, and $s$ the exponential factor lying between 0 and 1 . Neglecting the $d c \operatorname{part} \sigma_{d c}(T)$ in the above relation the value of $s$ can be determined from the linear slope of the logarithmic plot between $\sigma^{\prime}(\omega, T)$ and angular frequency $\omega$.

The $d c$ electrical conductivity $\sigma_{d c}$ is evaluated from $a c$ electrical conductivity data at lower frequency, the temperature dependent of $\sigma_{d c}$ can be expressed by the following Arrhenius equation $[12,13]$ :

$$
\sigma_{d c}=\sigma_{o} \exp \left(-\frac{E_{a}}{k T}\right)
$$

where $\sigma_{o}$ can be considered as the limiting value of conductivity at an infinite temperature, $E_{a}$ is the activation energy for conduction, $k$ is Boltzmann constant, and $T$ absolute temperature.

\section{EXPERIMENTAL DETAIL}

In the experiments Polyester was used as matrix material to prepare the polymer matrix composite. The rate of polymerization for this resin is too slow. For practical purpose the catalyst Methel Ethel Keton proxide MEKP in a proportion of $0.5 \mathrm{gm}$ for each $100 \mathrm{gm}$ of the resin, and accelerator Cobalt napthenate catalyst in a proportion of $0.1 \mathrm{gm}$ for each $100 \mathrm{gm}$ of the resin are used.

The sample of Polyester doped with $(0,6,12$, and 18$) \%$ of carbon black of thicknesses $\mathrm{d}=3 \mathrm{~mm}$ has been prepared using solution cast technique. After preparation of the resin with the desired volume fraction it was cast in the glass mould, and was left out in a dust free chamber for 24 hours at room temperature to complete setting. Finally, it was put in furnace at $60^{\circ} \mathrm{C}$ for four hours post curing.

In order to obtain a good contact for electrical measurements, the prepared samples of radius $1.5 \mathrm{~cm}$ have been coated with silver materials on both surfaces. The silver coated samples sandwiched between the two similar aluminum electrodes governed by a screw to minimize the parasite capacitance induced by the presence of air interstices at the interfaces between the sample and the electrodes. The whole assembly was placed in a temperature controllable isolated chamber, and the temperature was measured by Chromel-Alumel thermocouple using a digital multimeter with an accuracy of $\pm 1^{\circ} \mathrm{C}$. The thickness of the films was in the range of $(2.6-4.0) \mathrm{mm}$, it was determined using micrometer at different places in each film and an average was taken.

\section{RESULTS AND DISCUSSION}

The electrical measurements (dielectric constant $\varepsilon^{\prime}$, dielectric loss $\varepsilon^{\prime \prime}$ and $a c$ electrical conductivity $\sigma_{a c}$ ) of Polyester-carbon black composites of different carbon black contents were obtained at a frequency range $(0.5-$ $\left.10^{3}\right) \mathrm{KHz}$ and within the temperature range $(26-80)^{\circ} \mathrm{C}$. The measurements were carried out using Programmable Automatic Precision LCR meter type PM6036.

The variation of the dielectric constant and $a c$ electrical conductivity of the Polyester composite with respect to $w t \%$ of carbon black $(\mathrm{CB})$ to Polyester resin at $1 \mathrm{MHz}$ frequency, at different temperature $26,40,60$ and $80^{\circ} \mathrm{C}$ are shown in Fig. 1 and Fig. 2 respectively.

According to the obtained results, the dielectric constant shows weak dependent on $\mathrm{CB}$ contents up to $5 \% \mathrm{CB}$, and then start to increases in a large $\mathrm{CB}$ concentration, while the $a c$ electrical conductivity $\sigma_{a c}$ seems to be increased linearly with respect to the $\mathrm{CB}$ contents.

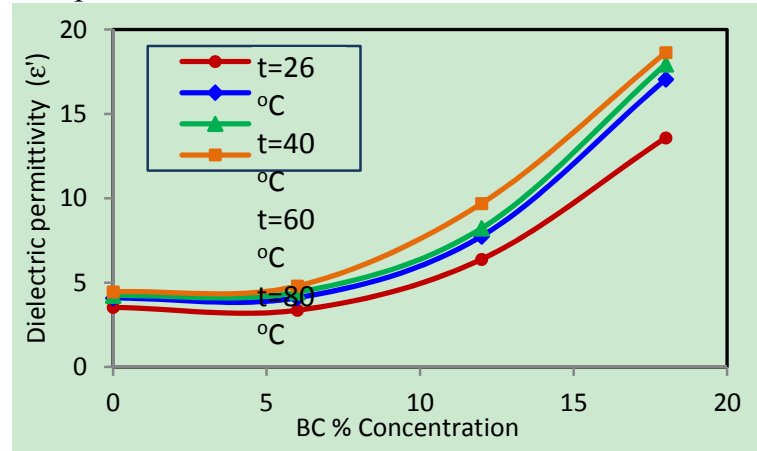

Fig. 1.Dielectric constant as a function of black carbon concentration for polyesters composite at different temperatures.

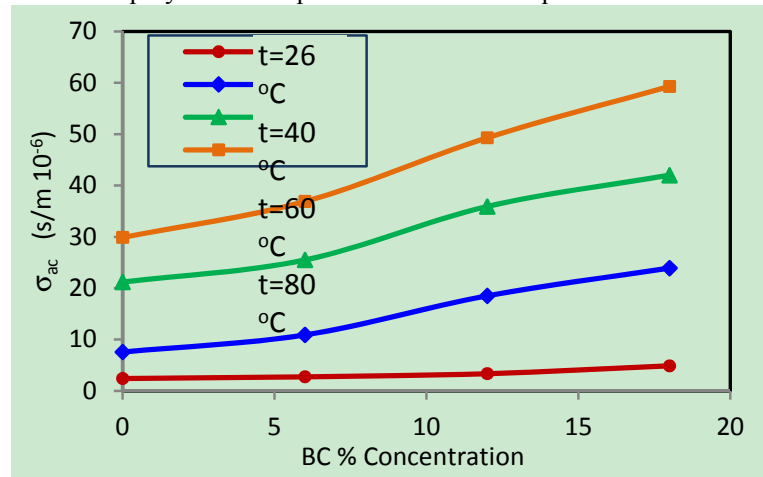

Fig. 2.ac electrical conductivity as a function of black carbon concentration for polyesters composite at different temperatures.

The increases of $\sigma_{a c}$ with temperature could be attribute to that more ions gained kinetic energy via thermally activated hopping of charge carriers, as well as the increment in temperature provides an increase in free volume and segmental mobility [16]. The increase of ac electrical conductivity with temperature, is more sensitive at higher temperature $\left(80^{\circ} \mathrm{C}\right)$ in comparison with room temperature. 
The dielectric constant $\varepsilon^{\prime}$ of Polyester reinforced with $18 \%$ of $\mathrm{CB}$ as a function of frequency at different temperatures was illustrated in Fig.3. It was observed that the dielectric constant decreases monotonically with increasing frequency and increases with increasing temperature. As well as, it can be noted at higher frequency the temperature effect is weaker in comparison with the temperature effect at lower frequency. The physics behind these variation can be explained depending on the fact that, in polar materials the initial value of dielectric constant is high, but as the frequency raised the value begins to drop which could be due lack of response of the dipoles, in other word incapability of the dipole to follow the field variations at high frequency [13].

In general, the $\varepsilon^{\prime}$ variation with temperature depends on the polymer types. For example in the case of nonpolar polymers the $\varepsilon^{\prime}$ is independent on temperature, while for strong polar polymers the dielectric constant increases with increasing temperature [17]. The increasing in $\varepsilon^{\prime}$ with temperature, especially at higher temperature in Fig.3, could be attributed to greater freedom of the molecular chain movement at high temperature [18], which lead to increase the polarization and hence dielectric constant is increased with increase of temperature.

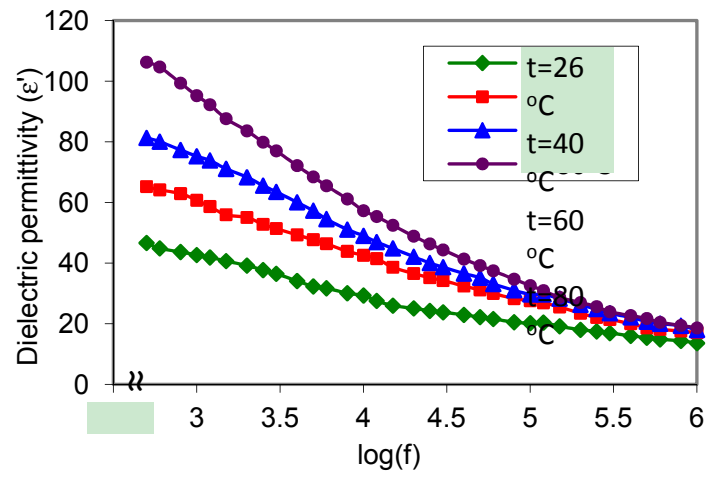

Fig. 3.Dielectric constant versus frequency at different temperatures of Polyester reinforced with $18 \%$ of Carbon black.

The series capacitance $\mathrm{C}_{\mathrm{s}}$ measured from RCL meter, is given by:

$$
C_{S}=C^{\prime}+\frac{1}{\omega^{2} R^{2} C^{\prime}}
$$

and the loss tangent by:

$$
\tan (\delta)=\frac{1+r / R}{\omega R C^{\prime}}+\omega r C^{\prime}
$$

where $r$ is small series resistance, and $C^{\prime}$ represent a frequency independent capacitive element which is connected in parallel with a temperature dependent resistive element $R$. The variation of dielectric constant $\varepsilon^{\prime}$ with frequency reflected the variation of $C_{s}$ through Eq. (2) when $A$ and $d$ of the samples are constant. According to Eq. (10) one can predict the decrease of $C_{s}$ with increasing $\omega$ eventually for $(\omega \rightarrow \infty)$ tending to stabilize at constant value of $C^{\prime}$ for all temperatures, on the other hand $C_{s}$ will increase with increasing temperature because of the decreasing value of $R$. The expression of $\tan (\delta)$ in Eq. (11) predicts a decrease in $\tan (\delta)$ with increasing $\omega$ where the term $\omega^{-1}$ is dominant for lower frequencies, followed by a loss minimum at $\omega_{\text {min }} \approx 1 /\left[C^{\prime}(r R)^{1 / 2}\right]$ [19] and finally the loss tangent tends to increase with $\omega$ above $\omega_{\min }$ where the $\omega$ term in the second part of the right hand of Eq. (11) is dominant.

The variation of $\tan (\delta)$ with frequency at various temperatures is represented in Fig.4. The decrease of $\tan (\delta)$ with frequency is clearly evident as predicted for low frequencies and tends to saturate at higher frequency, while no indication of a minimum is observed over the investigated frequency range. The higher the temperature the higher the loss tangent at lower frequency accompanies the less dependence on temperature at higher frequency. The increase in $\tan (\delta)$ with temperature is consistent with Eq. (11) as the $\omega^{-1}$ term becomes dominant due the decreasing value of $R$ with temperature, as shown in Fig.5. The resistance variation with temperature is clearly observed up to $100 \mathrm{KHz}$, then tends to stabilize at higher frequency, as a loss tangent (see Fig.4)

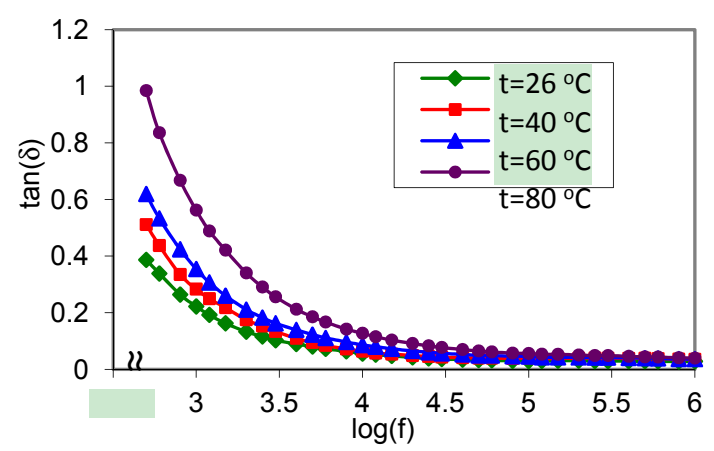

Fig. 4.Loss tangent versus frequency at different temperatures of Polyester reinforced with $18 \%$ of Carbon black.

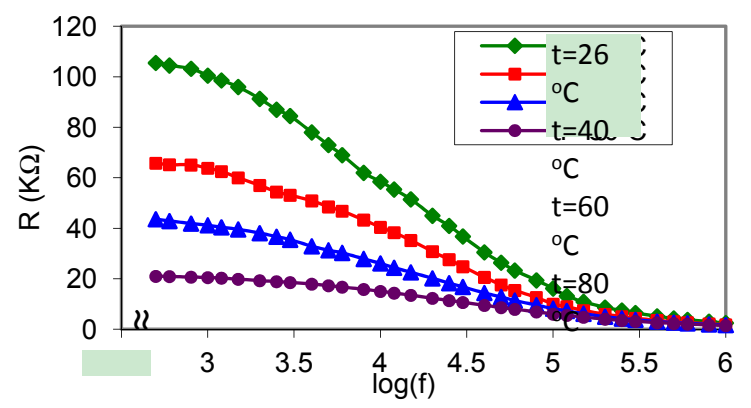

Fig. 5.Variation of resistance with frequency at different temperature of Polyester reinforced with $18 \%$ of Carbon black.

The frequency dependence of the $a c$ electrical conductivity $\sigma_{a c}$ for different values of temperatures is shown in Fig.6. According to the obtained results, $\sigma_{a c}$ increases with increasing temperature and frequency satisfying the Random Free Energy Barrier (RFEB) model, proposed by J.C. Dyre, which expects, at low frequency the conductivity will be constant and beyond a specified region of frequencies the conductivity strongly increases with frequency $[10,20]$.

Fig. 6 shows the linear trends between $\log \left(\sigma_{a c}\right)$ versus $\log (\omega)$. The electrical conductivity follows the universal power law represented in Eq. (8). The values of $s$ can be determined from the linear slope of lines in Fig.6. The parameter of $s$ as a function of temperature was tabulated in TABLE I.

The variation of parameter $s$ with temperature gives information on the specific mechanism involved. Depending on the obtained results $s$ is weakly decreasing function of temperature; this could be attributed that higher temperature, 
high mobility of free charges make them more frequency independent conductivity; as a result the $d c$ conductivity increases with increasing temperature [16].

TABLE I: EXPONENTIAL FACTOR VeRsus TEMPERATURE, For POLYESTER REINFORCED WITH $18 \%$ OF CARBON BLACK.

\begin{tabular}{cc}
\hline \hline Temperature $\left({ }^{\circ} C\right)$ & Exponential factor $s$ \\
\hline 26 & 0.77 \\
40 & 0.75 \\
60 & 0.70 \\
80 & 0.63 \\
\hline \hline
\end{tabular}

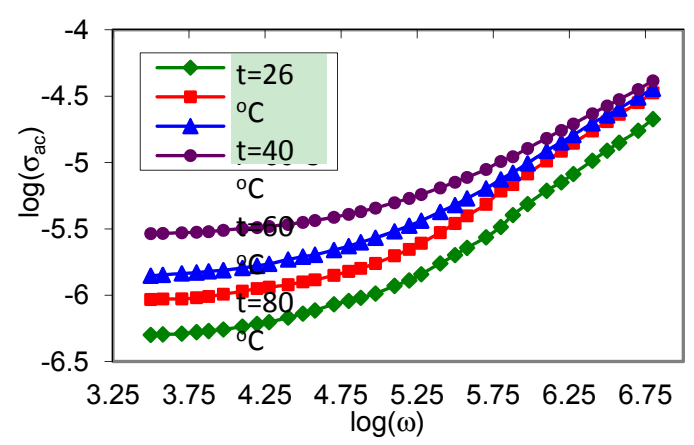

Fig. 6.Dependence of frequency on the ac conductivity at different temperatures of Polyester film doped with $18 \%$ of Carbon black.

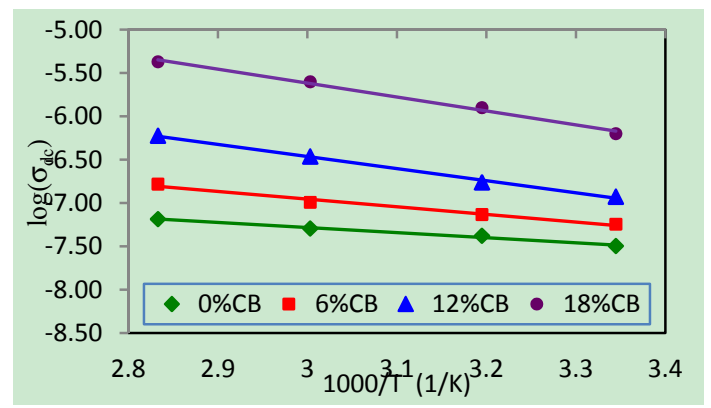

Fig. 7.Semilogarithmic plots of dc conductivity against reciprocal of temperature at different Carbon black concentrations.

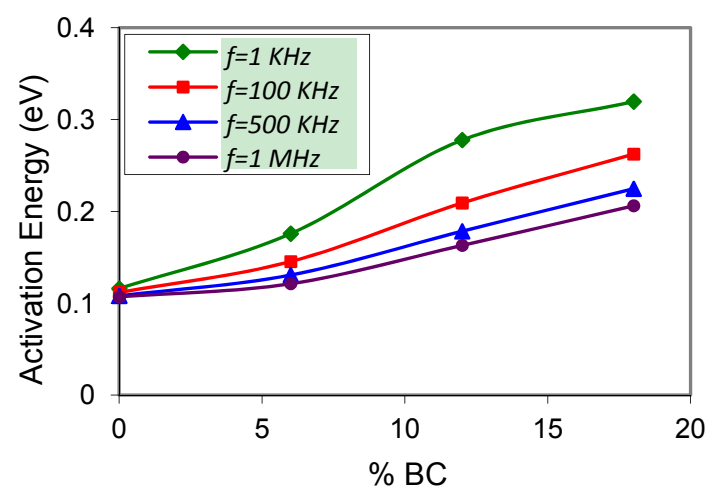

Fig. 8.Activation energy against Carbon black concentrations, for different frequency.

Fig.7 shows the relation between $\log \left(\sigma_{d c}\right)$ and $1000 / T$ for different $\mathrm{CB}$ concentrations, the solid line represent the best-fitted value to obtain slope. According to Eq. (9), and from the slope of parallel straight line, the activation energy $E_{a}$ is calculated, for a temperature range $(26-80)^{\circ} \mathrm{C}$. Fig. 8 , shows the variation of $E_{a}$ as a function of black carbon concentrations, for different frequencies. The estimated values of $E_{a}$ are essentially dependent on black carbon concentrations, and frequency.

It was clear that activation energy values increases with increasing the $\mathrm{CB}$ content, and decreases with frequency. The activation energy $E_{a}$ increases from $0.116 \mathrm{eV}$ for pure polyester to $0.319 \mathrm{eV}$ for $18 \% \mathrm{CB}$. The addition of $\mathrm{CB}$ to Polyester host enhances the electrical conduction of Polyester host due to the electronic and impurity contributions arising from the $\mathrm{CB}$ [9].

\section{CONCLUSIONS}

The temperature and frequency dependence of dielectric constant and $a c$ electrical conductivity of Polyester-carbon black had been carried out. The dielectric constant was found to be decrease with increasing frequency and increase with increasing temperature. This behavior was attributed to the polar nature of the polyester. The $a c$ electrical conductivity increases with the increment of temperature and it obeys universal power low. Increasing of the values of the electrical conductivity was driven by the mobility of free charges as temperature is increased. The variation of frequency exponential factor $\mathrm{s}$ between 0.63 and 0.77 , indicates a dominant hopping process at low temperatures. The increase of activation energy was observed with carbon black concentrations.

The second observation in this study worth to be mentioned relates to the relative value of electrical conductivity versus the carbon black contents; it was observed that even low amount of carbon black able to maximize the conductivity of the composite, we conclude that polyester carbon black composite is a good candidate and alternative way for obtaining conducting organic composites at low cost.

\section{REFERENCES}

[1] A. El-Khodary, A.H. Oraby, and M.M. Abdelnaby, "Characterization, electrical and magnetic properties of PVA films filled with $\mathrm{FeCl}_{3}-\mathrm{MnCl}_{2}$ mixed fillers", Journal of Magnetism and Magnetic Materials 320, 1739-1746 (2008).

[2] R.J. Sengwa, and S. Sankhla, "Characterization of ionic conduction and electrode polarization relaxation processes in ethylene glycol oligomers", Polymer Bulletin 60,689-700 (2008).

[3] T. Kimura, and M. Kajiwara, "Electrical properties of poly(n-butylamino) (di-allylamino) phosphazene", Journal of Material Science, 33(11), 2955-2959 (1998).

[4] S. Shekhar, V. Prasad, and S.V. Subramanyam, "Structural and electrical properties of composites of polymer-iron carbide nanoparticles embedded in carbon", Materials Science and Engineering B 133, 108-112 (2006).

[5] F. Carmona, and J. Ravier, "Electrical properties and mesostructure of carbon black-filled polymers", Carbon 40, 151-156 (2002).

[6] Woo Seok Chin, and Dai Gil Lee, "Dielectric Characteristics of E-glass-Polyester Composite Containing Conductive Carbon Black Powder", Journal of Composite Materials 41, 403-417 (2007).

[7] D.K. Das-Gupta, "Polyethylene: structure, morphology, molecular motion and dielectric behavior", IEEE Electrical Insulation Magazine 10(3), 5-15 (1994)

[8] F.U.Z. Chowdhury, and A.H. Bhuiyan, "Dielectric properties of plasma-polymerized diphenyl thin films", Thin Solid Films 370, 78-84 (2000).

[9] Ayman S. Ayesh, "Electrical and optical characterization of PMMA doped with $\mathrm{Y}_{0.0025} \mathrm{Si}_{0.025} \mathrm{Ba}_{0.9725}\left(\mathrm{Ti}_{(0.9)} \mathrm{Sn}_{0.1}\right) \mathrm{O}_{3}$ ceramic", Chinese Journal of Polymer Science 28(4), 537-546 (2010).

[10] Jeppe C. Dyre and Thomas B. Schrøder, "Universality of ac conduction in disordered solids", Reviews of Modern Physics 72 (3), 873- 892 (2000).

[11] J. Bisquert and G. Garcia-Belmonte, "Interpretation of AC Conductivity of Lightly Doped Conducting Polymers in Terms of Hopping Conduction", Russian Journal of Electrochemistry, 40(3), 352-358 (2004).

[12] S.P. Mondal, R. Aluguri, and S.K. Ray, "Dielectric and transport properties of carbon nanotube-CdS nanostructures embedded in 
polyvinyl alcohol matrix", Journal of Applied Physics 105, 114317 (2009).

[13] V. Raja, A.K. Sharma, and V.V.R. Narasimha Rao, "Impedance spectroscopic and dielectric analysis of PMMA-CO-P4VPNO polymer films", Materials Letters 58, 3242-3247 (2004).

[14] G.E. Pike, "ac Conductivity of scandium oxide and a new hopping model for conductivity", Physical Review B 6 (4), 1572-1580 (1972).

[15] F.M. Reicha, M. Ishra, and M.M. Gabr, "On the physical and electrical properties of poly propenal prepared by electropolymerization technique", J. of Phys. and Chem. of Sol. 64, 1157-1161 (2003).

[16] M.H. Harun, E. Saion, A. Kassim, M.Y. Hussain, I.S. Mustafa and M.A.A. Omer, "Temperature dependence of ac electrical conductivity of PVA-PPy- $\mathrm{FeCl}_{3}$ composite polymer films", Malaysian Polymer Journal 13 (2), 24-31 (2008).

[17] F.U.Z. Chowdhury, and A.H. Bhuiyan, "Dielectric properties of plasma-polymerized diphenyl thin films", Thin Solid Films 370, 78-84 (2000).

[18] M. Akram, A. Javed, and T.Z. Rizvi, "Dielectric properties of industrial polymer composite materials", Turk J. Physs. 29, 355-362 (2005).

[19] S. Darwish, "Capacitance measurements and ac conductivity of Nickel Phthalocyanine films", International Journal of Pure and Applied Physics 1(2), 165-172 (2005).

[20] Jeppe C. Dyer, "The random free-energy barrier model foe ac conduction in disordered solids", J. Appl. Phys. 64 (5), 2456-2468 (1988).

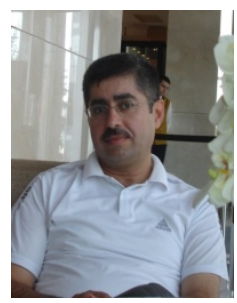

Omed Gh. Abdullah, was born in Sulaimani, Kurdistan Region-Iraq, on July 1969, He received the M.Sc degree in material science from Baghdad University, Iraq in 1999; and $\mathrm{PhD}$ degree in Material Science from Baghdad University, Iraq in 2006 . He is worked as a lecturer in the Salahaddin University-Erbil, and University of Sulaimani. Now he is a lecture in department of physics, Faculty of Science and Science Education,

University of Sulaimani, Kurdistan region-Iraq. His principle research interests have been in the fields of solid state physics, material science, and powder technology. His interests include fabrication and characterization of thin films, Physical properties of composite material, and simulation of Powder compaction. He is author or coauthor of 16 publications in refereed journals. He is a member of IACSIT, Iraqi Physics and Mathematics Society, Kurdistan Physicists' Syndicate, and Kurdistan Chemists \& Physists Society.

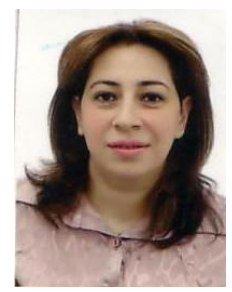

Gelas M. Jamal, was born in Baghdad-Iraq, on 1973, she received the M.Sc degree in laser physics from Baghdad University, Iraq in 1998; and PhD degree in Material Science from Baghdad University, Iraq in 2008. She is worked as a lecturer in the Baghdad University, and University of Sulaimani. Now she is a lecture in department of physics, Faculty of Science and Science Education, University of Sulaimani, Kurdistan region-Iraq.

Her research interests have been in the fields of laser interaction with silicon solar cells, and mechanical properties of composite material. She is a member of Kurdistan Chemists \& Physists Society.

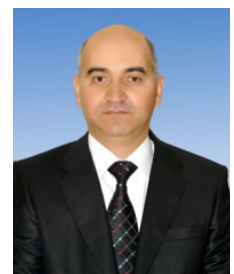

Dana A. Tahir, was born in Sulaimani, Kurdistan Region-Iraq, on 1968, He received the M.Sc degree in solid state from Salahaddin University, Iraq in 1995; and $\mathrm{PhD}$ degree in solid state from University of Sulaimani, Iraq in 2007. He is worked as a lecturer in the Salahaddin University-Erbil, and University of Sulaimani. Now he is a head of physics department, Faculty of Science and Science Education,

University of Sulaimani, Kurdistan region-Iraq. His research interests was Material Science, Oxide Glasses, Polymer Materials and Thin Film Deposition. He is a member of Kurdistan Chemists \& Physists Society.

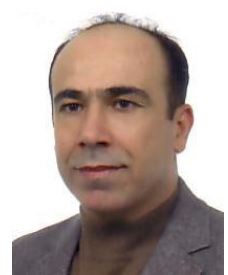

Salah R. Saeed, was born in Sulaimani, Kurdistan Region-Iraq, on September 1966, He received the M.Sc degree in solid state physics from Salahaddin University, Iraq in 1992; and PhD degree in Atomic physics from University of Jagiellonian, Poland in 2008. He is worked as a lecturer in the Salahaddin University-Erbil, and University of Sulaimani. Now he is a dean of Education College,

University of Sulaimani, Kurdistan region-Iraq. His research interests have been in the fields of nanostructure, surface composition modification, nano tribulogy, friction force. He is a member of Physics and Mathematics Society, Iraqi Physics and Mathematics Society, and Kurdistan Chemists \& Physists Society. 